\title{
A Supersonic Plasma Jet Source for Controlled and Efficient Thin Film Deposition
}

\author{
Ilaria Biganzoli ${ }^{1}$, Francesco Fumagalli ${ }^{1}$, Fabio Di Fonzo ${ }^{2}$, Ruggero Barni ${ }^{1}$, Claudia Riccardi ${ }^{{ }^{*}}$ \\ ${ }^{1}$ Dipartimento di Fisica Occhialini, Università degli Studi di Milano-Bicocca, Milano, Italy \\ ${ }^{2}$ Center for Nano Science and Technology, Italian Institute of Technology, Milano, Italy \\ Email: ${ }^{*}$ claudia.riccardi@mib.infn.it
}

Received August 29, 2012; revised September 30, 2012; accepted October 7, 2012

\begin{abstract}
A novel plasma source suitable for controllable nanostructured thin film deposition processes is proposed. It exploits the separation of the process in two distinct phases. First precursor dissociation and radical formation is performed in a dense oxidizing plasma. Then nucleation and aggregation of molecular clusters occur during the expansion into vacuum of a supersonic jet. This allows a superior control of cluster size and energy in the process of film growth. Characterization of the plasma state and source performances in precursor dissociation have been investigated. The performances of this new Plasma Assisted Supersonic Jet Deposition technique were demonstrated using organic compounds of titanium to obtain $\mathrm{TiO}_{2}$ thin nanostructured films.
\end{abstract}

Keywords: Plasma Sources; ICP Discharges; PECVD; Plasma Diagnostics; Titanium Dioxide; Thin Film Deposition

\section{Introduction}

The deposition of thin films using physical vapour deposition or chemical vapour deposition (CVD) techniques is a process that has many applications in material science [1]. In particular the production of nanostructured materials is, currently, one of the fields of more intense research and technological development. Thin films made of oxides, semiconductors or metals are especially interesting in this respect. The possibility to change their morphology at the nanoscale makes them suitable for applications like catalysis and photocatalysis, energy conversion or storage, rather than more conventional uses as barrier or protective coatings [2]. Despite their interest, few innovative techniques exists for the controlled deposition of nanoscale assembled materials. As an example, titanium and zinc oxides are usually produced by sol-gel synthesis of nanoparticles followed by sintering, generally yielding disordered films either with moderate porosity or in aerogel form $[3,4]$. Ordered structures, with a somewhat controlled porosity, have been obtained by using templating agents [5], even if their removal may cause some dimensional changes. Template-free fabrication and photocatalytic activity of hierarchically organized nanostructured $\mathrm{TiO}_{2}$ has been reported, by typical thin film deposition techniques, such as CVD [6,7], sputtering [8] and pulsed laser deposition (PLD) [9]. In particular enhanced surface reactivity was related to the

${ }^{*}$ Corresponding author.
3D columnar structure increasing the surface area, controlled by their thickness and spacing [6]. Other notable examples of 3D film structure were reported in the form of fractal "forest-like" $\mathrm{TiO}_{2}$ deposit by CVD [10]. In general, however, the active surface area of these films is limited and hardly controllable to a large extent and there is only a modest control over the deposited material composition. Even PLD, though being a versatile, one-step, template-free process [9], still has limited chances of upscalability, needed in large area applications such energy. Plasma enhanced CVD (PECVD) is a particular version of thin film deposition. In the field of nanostructured materials it was pioneered by applications to $\mathrm{Si}$ and $\mathrm{Ge}$ quantum dots production, using silane injection in an argon plasma [11]. Although promising, no examples of nano-assembled functional films have been demonstrated so far. Moreover, there are very few examples of plasma processes capable of depositing nanostructured films with controlled chemistry, morphology and porosity [12]. The novelty of the proposed approach, which we denominated Plasma Assisted Supersonic Jet Deposition (PASJD), is the segmentation of the gas phase material synthesis in two separate steps: chemistry control in a reactive cold plasma environment; nucleation and assembling control by means of a supersonic inseminated jet over a substrate. High throughput and fast deposition rates could be achieved using high density plasmas. Vaporizable and stable monomer could be employed as the source of the oxides, the semiconductors or even the me- 
tals. The extraction of a supersonic plasma jet allows to focus the precursor flow on the substrate and to control the growth and the sizes of clusters. Over conventional gas phase processes the proposed technique allows control of the kinetic energy of clusters, whence control on their assembly pattern on the substrate. In particular when molecular cluster deposition is performed under condition of supersonic jet several advantages could be envisaged including the better collimation and stability and the control of crystallinity. By changing the conditions during processing it is also possible to obtain graded films. Here we present results concerning the design and performances of a novel plasma source with a reaction chamber where plasma dissociation and clusterization of precursors could be achieved and from which a sample of reactive radicals could be extracted. Extraction is performed through a nozzle, producing a supersonic jet which propagates in an expansion chamber where deposition processes happen, to be used to grow thin films with controlled morphology at high yield. We will characterize the plasma in our source and then we will demonstrate that ordered nanostructured films of $\mathrm{TiO}_{2}$ could be grown and that their morphological properties, similar to dendritic structures obtained by means of PLD, can be tuned, by varying the process parameters. Then a fair parameter control could be achieved in order to tailor the optoelectronic properties of synthesized material.

\section{The Experimental Setup}

As stated in the introduction, our main purpose was to spatially separate the region where dissociation of the precursor is achieved in a dense plasma environment and another region where deposition takes place, on a substrate which is exposed to a flow of mainly neutral particles and clusters. A sketched view of the source setup is shown in Figure 1. As for the electrical discharge our approach was to use an inductively coupled plasma (ICP) $[13,14]$. This allows to reach quite large plasma densities and relatively small electron temperatures at moderate pressures, $1 \div 100 \mathrm{~Pa}$. This favours dissociation of external, weakly bound chemical groups in organic precursors, partially preserving the backbone structures, for instances $\mathrm{Si}-\mathrm{O}$ bonds in HMDSO [15] or Ti-O ones in titanium ethoxide [16]. A radiofrequency (RF) signal was then applied to an antenna facing the plasma chamber. For the discharge feeding we employed a commercial RF power supply (PFG-1600 by Huettinger, maximum power $1.6 \mathrm{~kW}$ ). Then our experiments have been performed all at a fixed frequency of $13.56 \mathrm{MHz}$. An L-type matching box was used along the transmission line. A passive system fits our needs, since plasma showed to be quite stable during operation, even during the fairly long depo-

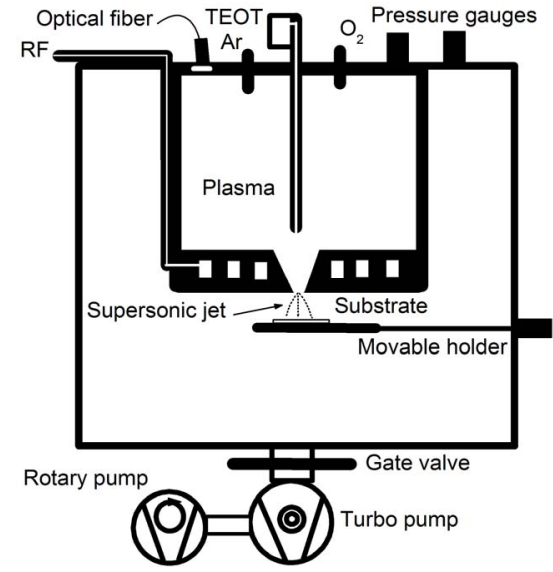

Figure 1. Lay-out of the experimental setup.

sitions exceeding 30 minutes. To reduce cable length, the system was mounted directly on top of the vacuum chamber and connected with the RF antenna through copper straps (see Figure 1). The measured reflected power was below $1 \%-2 \%$ even at power level of about $500 \mathrm{~W}$. We have used a two and three quarters loop planar antenna made of hollow copper wire (4-external, 3internal $\mathrm{mm}$ diameters). This allows to circulate deionized water for cooling purposes. The total length was about $80 \mathrm{~cm}$ with an outer and an inner radius of 54 and $40 \mathrm{~mm}$ respectively. The antenna was inserted in a teflon scaffold, a perforated disk $(22 \mathrm{~cm}$ diameter, $17 \mathrm{~mm}$ thick with a $33 \mathrm{~mm}$ radius hole), and voids were filled with an epoxy patch adhesive (see Figure 1). Over the internal side of the teflon scaffold, where the antenna is embed$\mathrm{ded}$, an alumina perforated disk (135 $\mathrm{mm}$ diameter, $2 \mathrm{~mm}$ thick) is placed, facing the plasma. This reduces contamination due to teflon sputtering. It also sustains much better the heat load from the plasma exposure. Under the teflon scaffold, an aluminium disk is placed, sealing the plasma chamber. In the central part of the disk, where there is the transition between the plasma and the deposition chambers, the nozzle region is excavated, as described below. The supply RF signal is connected to the external edge of the coil (and shielded), while the internal end is connected to the ground. The RF connections run down from the top of the device along the external walls of the plasma chamber (see Figure 1), to reduce contamination. The total inductance of the antenna system was measured to be $2.5 \mu \mathrm{H}$ with an impedance of about $213 \Omega$ at RF. Particular care was paid to the earthing. Large copper straps were employed to connect the different parts of the vacuum chamber (main body, top and bottom plates, internal plasma chamber) and the RF network to ensure low resistance, short ways to ground. This proved essential to prevent discharges happening at unwanted locations and RF power dispersions. Afterwards stable working conditions, even at relatively 
high power levels, could be maintained for times exceeding hour-long operations. Localized temperature measurements on the plate under the antenna confirm that heating is moderate even after running more than 1 hour at $500 \mathrm{~W}$. Our device operates under moderate vacuum conditions, that is roughly in a range $1 \div 100 \mathrm{~Pa}$. It was built up out of a commercial stainless-steel cylindrical vacuum chamber $(31 \mathrm{~cm}$ diameter and $40 \mathrm{~cm}$ height). Several ports allows to monitor the discharge as well the insertion of diagnostics or sample holders. On the bottom a large (100 mm diameter) vacuum flange constitutes the main connection to the pumping system. On the top flange 11 ports where placed, for gas/vapour-inlet, RF feed throughs, view ports and diagnostics. The plasma chamber is appended in the upper central part of the device. It is made of a thick aluminium cylinder $(125 \mathrm{~mm}$ diameter and $95 \mathrm{~mm}$ height) closed on the bottom by the antenna scaffold and the sealing aluminium disk. Its volume is approximately 1.15 liters. Although a vacuum proof insulation from the main chamber is not strictly needed, all junctions of the plasma chamber were sealed using Viton rings in order to prevent leakage of precursors and of reactive agents in other part of the device. The rest of the device constitutes the deposition chamber. Its volume is approximately 30 liters. However the active region is just below the plasma chamber. On a side port a rotable/movable vacuum feedthrough is used to move forward/backward the sample holder and to expose different substrates at fixed distances from the plasma chamber exit. The connection between the plasma chamber and the deposition chamber consists of a convergent nozzle followed by a thin aperture. In a first realization the latter consists of a slit (22 mm length, $2 \mathrm{~mm}$ width) cut in a $0.9 \mathrm{~mm}$ aluminium foil. The lay-out is sketched in Figure 2. A circular hole ( $7.5 \mathrm{~mm}$ diameter) was tried too, the better shape being determined by the deposition needed properties [17]. Above this orifice, the aluminium disk was excavated forming a gentle transition (convergent nozzle) from the teflon aperture, at the level of the

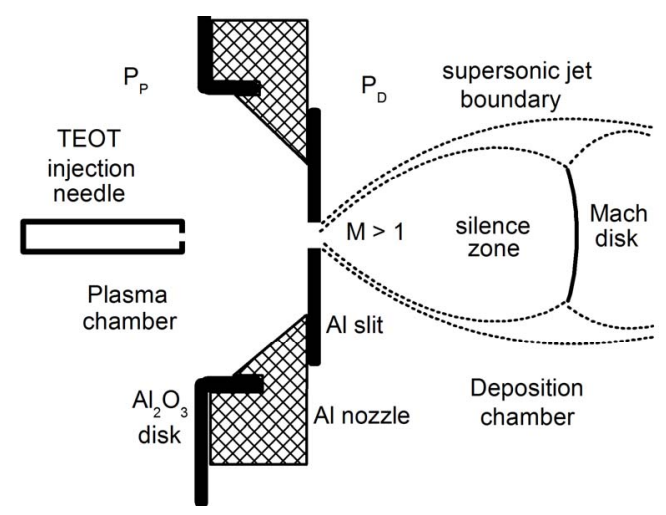

Figure 2. Cross section of nozzle region with a schematic view of the jet expansion in our experimental setup.
RF coil to the bottom of the disk aperture (a $22 \times 30 \mathrm{~mm}$ rounded rectangle), where one of the different apertures is to be sealed. With this choice, when a differential pressure exists the flow out of the plasma chamber assumes the form of a jet. In principle, other convergent-divergent configurations could be used (for instance De Laval nozzle [18]), but this one has the advantage that the aperture foil can be easily removed and modified. As we will discuss in a short while, when the pressure difference is high enough the jet expansion can become supersonic. We have already said that such a capability was one of the main goal to be achieved in the design of the source. In this first design of the source, we choose to use a single pumping system composed of a turbo molecular pump (Turbovac TW250-S by Leybold) and a rotary pump (Alcatel 2020A). As already mentioned, the pumping system is connected at the bottom of the main chamber, through a gate valve. The actual pressures in the two chambers is controlled by the conductances of the connections (see Figure 1) and by the position of the manual leakage valves connected to the gas-mixture feed system, as it will be discussed below. Three direct gas-inlet on the top plate of the device were used to prepare the gas phase in the plasma chamber. The central port was employed for the precursor injection system. Other two ports, $50 \mathrm{~mm}$ out of center, are used for the gases. Two micrometer dosing valves (EVN-116 by Pfeiffer) were chosen, in principle supporting an extended flowrate range of more than 8 order of magnitude, $\mathrm{Q}=10^{-6} \div 10^{2} \mathrm{~Pa} \cdot \mathrm{m}^{3} \cdot \mathrm{s}^{-1}$. In the experiments discussed below, an argon and oxygen mixture was chosen. Oxygen was selected as the oxidizing agent to chemically attack the organic groups in precursors. Oxygen is in fact the most efficient oxidizing plasma, but used as a pure gas has many drawbacks, including strong stresses of the pumping system and of the vacuum parts. Argon dilution was then used to provide a stable, not so stressing plasma environment. Argon ICPs are also well known in literature and their properties have been studied at length $[13,19]$. High purity argon and oxygen cylinders $(5.0$ by Sapio) were employed. A dedicated and calibrated, remote controlled flow-meter (EL-Flow by Bronkhorst) has been installed during the preliminary device test phase, with argon. Using such calibration, we could assess that during experiments flow-rate was changed in a somewhat limited range of $Q=0.01 \div 0.1 \mathrm{~Pa} \cdot \mathrm{m}^{3} \cdot \mathrm{s}^{-1}$. The nominal pumping speed $\mathrm{S}$ and the real ones of argon $\left(0.21 \mathrm{~m}^{3} \cdot \mathrm{s}^{-1}\right)$ and oxygen $\left(0.23 \mathrm{~m}^{3} \cdot \mathrm{s}^{-1}\right)$ are similar for our pump thus providing a sensible control of the gas-phase composition, preventing accumulation and a derive in the gas-phase mixing. Pressure during operation is provided by two capacitance gauges (an Alcatel ADS-1003, upper range of $1 \mathrm{kPa}$ in the plasma chamber and a Pfeiffer CMR264, upper range of $100 \mathrm{~Pa}$ in the deposition chamber). 
Their pressure reading is rather unsensible to the gasphase composition and their precision is good in the working pressure ranges. Then the argon/oxygen mixing ratio was simply evaluated by measuring the pressure ratio after opening at first argon dosing valve and subsequently oxygen one. To control contamination and overall purity, the system could be evacuated using the full capability of the pumping system. This is performed routinely before sample preparation is to be started. Vacuum pressure is controlled in the main chamber by a Penning gauge (PTR-90 by Leybold). Although the use of precursors in deposition processes makes unavoidable some contamination of the chamber walls, the quite large volume of the chamber and the flow of the gas somewhat limited to the central part of the device helps in keeping at acceptable levels. Periodic cleaning of the plasma chamber walls, which can be completely removed, is performed. Then outgassing flowrate could be controlled and vacuum levels of a few $10^{-4} \mathrm{~Pa}$ could be achieved after a few hours of full speed pumping, since sealing. For the injection of the precursor we have chosen a needle. While for the discharge ignition and development in the plasma chamber a uniform and well mixed gas mixture is a nice starting point, efficient precursor dissociation does not need it. Indeed a mixing of the precursor outside the plasma chamber could provide complications, since heating is useful to vaporize liquid compounds or to increase the vapour pressure of a precursor but it is not needed for a gas. A uniform distribution of the precursor in the whole plasma volume is not needed too, since deposition is performed elsewhere and most of the volume is too far from the injection point. Moreover the effective residence time in the plasma region needs to be optimized or, at least, controlled in each deposition process, and cannot fit a once and for all scheme as implied by performing the mixing of the gas-phase outside the plasma chamber. We used a hollow copper wire (6-external, 4-internal $\mathrm{mm}$ diameters) with a $1.5 \mathrm{~mm}$ hole drilled at the bottom end. The wire is mounted on a vacuum feedthrough at the center of the top plate and gets down in the plasma chamber to a regulable depth, in the experiments reported here $87 \mathrm{~mm}$, thus being a few $\mathrm{mm}$ above the level of the RF coil and $30 \mathrm{~mm}$ from the slit. Another micrometer dosing valve of the same kind controls the vapour flow. A reservoir, a sintered aluminum flask of a few tens of $\mathrm{ml}$ with a brass sealing is used to store liquid precursors. After refilling, the flask and the micrometer valve connections were briefly pumped by a rotary pump. This remove most of the residual air from the flask, allowing the reservoir to be stored with the liquid precursor in equilibrium with its saturated vapour. This obviously select as usable precursors only those with a sufficiently high vapour pressure at set temperature, otherwise outgassing and residual air will contami- nate the gas-phase. To increase the flow, heating of the precursor flask and the injection system constitutes the most viable way. A heater was then put under the flask. Insulation of the injection system is provided too and tested by the use of several thermocouples. It should be noted that the injection needle gets heated by the plasma and by the RF itself. So the actual temperature of the precursor vapour at the injection in the plasma is somewhat depending on the plasma conditions. However, after the opening of the dosing valve, equilibrium is reached quite fast and a steady operation condition could be established and maintained allowing long, even hour-long deposition processes. In order to avoid contaminations and not to perform deposition under a poorly controlled environment, sample holder was not exposed to the jet until such steady condition is reached. In the experiments discussed in the present paper we have employed as a precursor the titanium ethoxide (TEOT). It is widely used in $\mathrm{TiO}_{2}$ depositions [20]. It already bears the tetrahedral structure of the titanium oxide, while the lateral hydrocarbon chains are easily removed by oxygen in the plasma chamber. The effective interaction length of precursor molecules is determined by the position of injection point and by the plasma conditions, mainly the density of oxidizing agents. This could be tuned in order to produce an optimal flow of seed radicals at the nozzle, to fed the plasma jet. In this respect the injection system and the plasma source could be easily fitted for different kind of precursors. Even concerning only $\mathrm{TiO}_{2}$ depositions, we have demonstrated that titanium isopropoxide could be used instead of TEOT, obtaining similar satisfactory results. The actual pressures in the two chambers are controlled by the conductances of the connections shown in Figure 1, their values depending on the flowrate $Q$ determined by the leakage valves opening and by the pumping speed $S$. The pressures in the plasma chamber $P_{P}$ and in the deposition chamber $P_{D}$ are given by the following expressions [21]:

$$
\begin{gathered}
P_{P}=Q \cdot\left(1 / C_{N}+1 / C_{P}+1 / S\right) \\
P_{D}=Q \cdot\left(1 / C_{P}+1 / S\right),
\end{gathered}
$$

where $C_{N}$ and $C_{P}$ are the conductances separating the plasma from the deposition chambers and the deposition chamber from the turbo-molecular pump, respectively. The values of $\mathrm{S}$ and $C_{N}$ are in a sense fixed by the setup choice. On the contrary, $C_{P}$ could be varied by throttling partially the gate valve at the bottom of the main chamber, reducing the overall pumping speed. This also increases the pressure in the deposition chamber which, as discussed previously, is useful to enhance clusterization and to grow porous films with a structure at the nanometer scale [9]. Q can also be easily changed regulating the opening of the micrometer valves. From the operating 
point of view, at first the overall pumping speed is reduced by throttling partially the gate valve at the bottom of the main chamber. Then the chosen pressures of argon are established, by opening the dosing valve and letting the system reach equilibrium. Afterwards the oxygen valve is opened and regulated until the desired pressures are reached at equilibrium. Normally plasma is switched on before precursor is injected. Power is raised gradually, in order to let the system heat and reach an equilibrium. Only afterwards the injection valve is opened and the precursor is let enter the plasma chamber. Again a few minutes are needed before a steady plasma and pressure is established in the device. Then deposition processes can start, moving the sample holder in the desired position and exposing the substrate to the jet coming from the nozzle. Although roughly, the value of the conductances could be estimated, in the different pressure ranges and could be used to estimate residence times as well as the relative weight of diffusion and flowrate of the species in the device. The pressure range we are interested in concerning the deposition chamber is $0.1 \div 10 \mathrm{~Pa}$, preferably above $1 \mathrm{~Pa}$, which is a compromise among the needs of a pressure not too high for the turbomolecular pump to work well, sufficient to control the clusterization, without affecting the capability of reaching supersonic expansion. Consequently in the plasma chamber, the pressure ranges between $1 \div 100 \mathrm{~Pa}$. This turns out to fit nicely the maximum coupling efficiency of radiofrequency discharges [14]. We have evaluated the Knudsen number $K n$ for argon and oxygen at room temperature as a function of both the pressure and the characteristic length. $K n$ value gives an indication of the flow type at low pressure: molecular $(M)$ if $K n>0.5$, transitional $(T)$ if $0.01<K n<0.5$, viscous $(V)$ if $K n<0.01$ [21]. It turns out that our flow regime in the deposition chamber will be mainly a transitional one. In the plasma chamber it is well within the viscous flow regime both for argon and oxygen. Only below $7 \mathrm{~Pa}$, the plasma gas phase enters into the transition regime. It is also worthy to notice that the flow is generally transitional in the nozzle region where precursor is injected. In addition well mixed reactor conditions are fulfilled in the whole pressure range in the plasma chamber [22]. Even more so happens in the deposition chamber, but in general deposition is performed near or within the Mach disk, to take advantage from the supersonic jet expansion. The same applies, albeit marginally in the nozzle region, where the precursor quickly mixes up in the plasma and reacts repeatedly, before being ejected in the plasma jet. The ratio $\mathrm{R}$ of the pressures in the two parts of the device is controlled by the $C_{N}$ conductance value, which is dominated by the rectangular slit, respect to the effective pumping speed. This ratio is a very important parameter since it determines whether the flow becomes supersonic after the nozzle. An appro- ximate formula for the conductance of a thin slit in a transitional flow was proposed in reference [23]. Together with the correction for the finite thickness of the slit [24], this could be used to calculate a value of $C_{N}=$ $(5.5 \pm 0.1) \times 10^{-3} \mathrm{~m}^{3} \cdot \mathrm{s}^{-1}$, strictly valid for $R>10$ and $K n<$ 0.1 . This is in fair agreement with the estimate made from the experimental measurements taken with an argon flow, using the calibrated flowmeter and the pressure readings in both chambers, $C_{N}=(5.6 \pm 0.3) \times 10^{-3} \mathrm{~m}^{3} \cdot \mathrm{s}^{-1}$. Given this conductance, the ratio $\mathrm{R}$ can reach a maximum equal to 38 in argon, largely sufficient for a supersonic jet expansion. Indeed in order to have this $R \sim 2$ is enough (more precisely $R=2.05$ for argon), as it could be demonstrated under the approximation of considering a convergent nozzle whose section decreases progressively and isentropic flow [18]. Here Mach number $M=$ 1 is reached at the nozzle exit. Corrections due to the sharp edge thin orifice like ours are small. For instance, with a circular orifice $M=1$ on the axis is reached approximately 0.25 diameters downstream respect to the geometrical exit [25]. For $R>2$ the flow dynamic in the nozzle region is unaffected by the background pressure in the deposition chamber (such case is thus named chocked nozzle). So, for instance, the local pressure at the nozzle exit is about $P_{P} / 2$, independently of the pressure $P_{D}$. The flow there is underexpanded and continues its expansion, thus reaching supersonic speeds. In this process the pressure drops below $P_{D}$, until this boundary condition is re-established through compression shock waves, which consist in thin regions where strong gradients of pressure, temperature and velocity occur. So, the expansion region constitutes a sort of plume emerging from the nozzle, laterally limited by oblique shocks and downstream restricted by the so called Mach disk shock. This region is often called zone of silence, being unaffected by the background pressure [18]. For a Mach disk with limited lateral extension we can assume that the wavefront is perpendicular to the flow direction, that is to say that we have a normal shock, for which the so called RankineHugoniot relations hold, connecting flow properties upstream and downstream the discontinuity [18]. In first approximation the deposition chamber conditions are completely re-established through a single shock, so the same background pressure $P_{D}$ is established downstream the Mach disk as well as in the rest of the deposition chamber. In the zone of silence the isentropic approximation is still adequate [18]. To further simplify, one can notice that a few diameters from the nozzle exit the flow field gets similar to that generated by a point source placed in the nozzle throat: streamlines are almost straight and radiates from the throat, velocity raises, and density decreases along the streamline as the inverse square of distance. Evidence in literature shows that such approximation is sensible, since the exit geometry (noz- 
zle versus orifice, sharp-edges, slit vs orifice) has only a limited influence on the central streamline properties [25]. To have an order of magnitude estimate here we report the solution for the Mach number $M$ and the pressure $P$ at a downstream distance $x$ from the source along the centerline, in case of a circular orifice with diameter $D$ [26]:

$$
\begin{aligned}
& M(x)=A \cdot\left(\frac{x-x_{1}}{D}\right)^{\gamma-1}-\frac{1}{2}\left(\frac{\gamma+1}{\gamma-1}\right) /\left[A \cdot\left(\frac{x-x_{1}}{D}\right)^{\gamma-1}\right], \\
& \frac{P(x)}{P_{p}}=A^{-2 /(\gamma-1)} \cdot\left(\frac{\gamma+1}{\gamma-1}\right)^{\gamma /(\gamma-1)}\left(\frac{\gamma+1}{2 \gamma}\right)^{1 /(\gamma-1)}\left(\frac{x-x_{2}}{D}\right)^{-2},
\end{aligned}
$$

with $A=\{3.26 ; 3.65\}, x_{1}=\{0.075 \mathrm{D} ; 0.4 \mathrm{D}\}, x_{2}=\{0.04 \mathrm{D}$; $0.13 \mathrm{D}\}$ for $\gamma=\{1.667 ; 1.4\}$. In the zone of silence the Mach number progressively increases with the distance from the nozzle exit. This particularity allows to select the velocity of particles and thus to grow films with different characteristics. The pressure ratio $R$ controls the Mach disk position, and thus the maximum speed achievable. With our setup Mach numbers up to 8 can be obtained. It could be noticed that another way for controlling the cluster kinetic energy consists in modifying the orifice shape. In general we could observe that within this supersonic expansion region, the reduced number of collisions does not favor cluster nucleation, so the particles available for deposition are mainly monomers and small clusters. Consequently, the zone of silence allows to synthesize films with small nanostructures and grains. On the contrary, since pressure rises in correspondence of shock waves, the deposition of clusters with higher sizes and lower kinetic energies can be performed outside of the silence zone. As for the Mach disk, this is located at a distance $X_{M O}$ for a circular orifice with diameter $D$ and at $X_{M S}$ for a slit with aspect ratio $w / h>1$, where $w$ and $h$ are the slit width and height respectively [27], while its lateral extension $D_{M O}$ can be estimated according to [28]:

$$
\begin{gathered}
X_{M S} / h=(w / h)^{0.47} \cdot\left(X_{M O} / D\right), \\
X_{M O} / D=0.67 \cdot \sqrt{R}, \\
D_{M O} / D=0.36 \cdot R^{0.6}-0.59 .
\end{gathered}
$$

Such formulas predict that the Mach disk location and lateral extension increase with $R$. The Mach disk turns out to be about $7 \mathrm{~mm}$ from the nozzle at $R=2.05$, up to $31 \mathrm{~mm}$ at the highest $R$ in our setup. Mach disk lateral extension was assessed too, increasing up to $30 \mathrm{~mm}$ within the whole $R$ range. This gives us room to work within a somewhat extended area even inside the silence zone where deposition could be performed.

\section{Diagnostics}

The electrical characteristics of the discharges have been measured by means of probes located outside the vacuum chamber. A high voltage probe (Tektronix P6015A, granted for a bandwidth of $75 \mathrm{MHz}$ ) was chosen for monitoring the voltage signal sent through the antenna and was connected at the copper strap-vacuum feed-through junction on the top of the device. The probe was calibrated and the tuning was optimized to enhance sensitivity near the RF frequency. Calibration factor was $1550 \pm 100$, with a phase delay $1.78 \pm 0.13 \mathrm{rad}$. Besides this, we have taken some measurement using a current probe. This consists of a home-made Rogowski coil which was developed to study fast microdischarges in atmospheric pressure plasmas [29]. It has enough sensitivity and the wide bandwidth necessary for use at RF. The calibration factor at such frequency was $5.67 \pm 0.01 \mathrm{~A} \cdot \mathrm{V}^{-1}$, with a phase delay $-0.144 \pm 0.013 \mathrm{rad}$. This allowed us to measure current and voltage signals with a digital oscilloscope (Tektronix TDS-5104) with sampling rate equal to $1.25 \mathrm{GSa} / \mathrm{s}$ and $1 \mathrm{M} \Omega$ input impedance. Optical emission spectroscopy (OES) can be employed to reveal the light emitted by the de-excitation of electronic energy levels of atoms, molecules, ions and radicals which have previously been excited in the discharge. Since in the cold plasma environment typical of ICP the excitation process happens mainly by impacts with plasma electrons, the emission spectra could be used also to extract information about their density or energy [30]. From the intensity of emission lines with different wavelengths one can identify chemical species as well as gain insight about their abundances and those of the exciting agents. We have analyzed the discharge emission spectra by means of a wide band, low resolution spectrometer (AvaSpec-2048 by Avantes) equipped with a $10 \mu \mathrm{m}$ slit, a holographic grating (300 lines $/ \mathrm{mm}$, blazed at $300 \mathrm{~nm}$ ), a coated quartz lens to increase sensitivity in UV and a 2048 pixels CCD. The spectrometer has a resolution of about $0.8 \mathrm{~nm}$ and a spectral band extending from $180 \mathrm{~nm}$ to $1150 \mathrm{~nm}$. Emission spectra of the discharges have been recorded directly imaging the plasma region through a quartz view-port on the top flange with an UV enhanced optical fiber. Thanks to its aperture the effective view-field covers most of the discharge volume. The system made up by the optical fiber, the monochromator and the CCD has been previously calibrated with both a deuterium and halogen lamp (Avalight-DHc by Avantes). This allows to correct for the device sensitivity and to obtain the real relative intensities of emission lines at different wavelengths. The exposure time has been optimised to match the CCD sensitivity, avoiding overcounts. During all the experiments, a large number of spectra have been acquired and averaged for noise reduction. 
Dark spectra with comparable statistics were acquired before each experiment and have been subtracted afterwards. Although somewhat outside the scope of our paper, we have also investigated thin film morphology and composition. Deposited films were analyzed with an FE-SEM (Zeiss Supra 40), equipped with an Oxford EDS analyzer. Their chemical composition was then measured with a precision of about $1 \%$.

\section{Experimental Results}

As it is well known ICP discharges operates in two different regimes, so-called E- and H-mode [31]. The names refer to the main actor sustaining the plasma state, being in the former case the electrostatic electric field provided by the voltage applied to an edge of the radiofrequency antenna, while in the latter the oscillating magnetic field locally inducing the electric field needed to sustain the plasma. Only in the second case the name ICP refers actually to the prevailing impedance of the antenna plasma coupling. By rising incrementally the voltage applied to the antenna through the matching network, the gas-phase undergoes an electrical breakdown and usually the ignited discharge operates in the E-mode. Increasing the voltage, a sharp transition occurs and the discharge enters the H-mode [32]. Apart from the geometry of the RF antenna and of the plasma chamber, the minimum voltage needed to enter the H-mode is determined by the ratio between the radiofrequency $\omega$ and the momentum transfer collision frequency which is controlled by the gas pressure [31]. As discussed above, the relevant pressure range in the plasma chamber will be $10 \div 100 \mathrm{~Pa}$. Initially plasma was produced in argon alone. Breakdown happens at a low power level $(10-20 \mathrm{~W}$ in the pressure range) after suitable RF-line adapter conditions are chosen and fixed to optimize network matching. The HV signal measured by the probe at the different power levels is shown in Figure 3. The steep rise at low power is due to the E-mode, with voltage proportional to the square root of the power. The voltage drops, even by a few hundreds volts, when the regime transition starts and the coupling is changing. The transition is somewhat smooth happening over a quite extended range of power, $30-60 \mathrm{~W}$, which is larger the lower the pressure. This is in agreement with similar observations published in literature [33]. After the H-mode is fully established, the HV starts rising linearly with the power level, at least in the limited range considered. From the simultaneous measurement of the current and voltage signals it is possible to calculate the values of the impedance of the antenna-plasma load. The same measure could be extracted form the values fixed for the capacitances of the RF matching box, when the best tuning conditions are reached [14]. This is displayed in Figure 4. The two

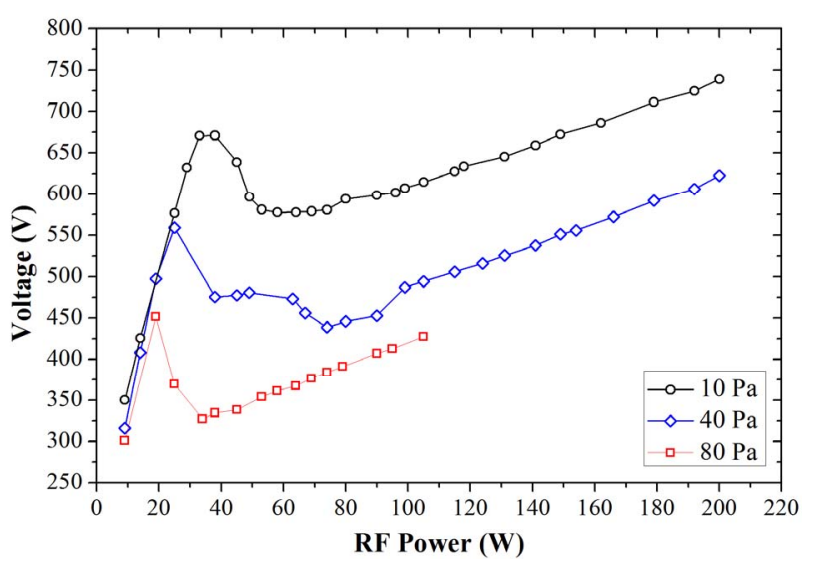

Figure 3. Coil HV amplitude as a function of the RF power level in argon discharges at pressures of 10, 40 and $80 \mathrm{~Pa}$.

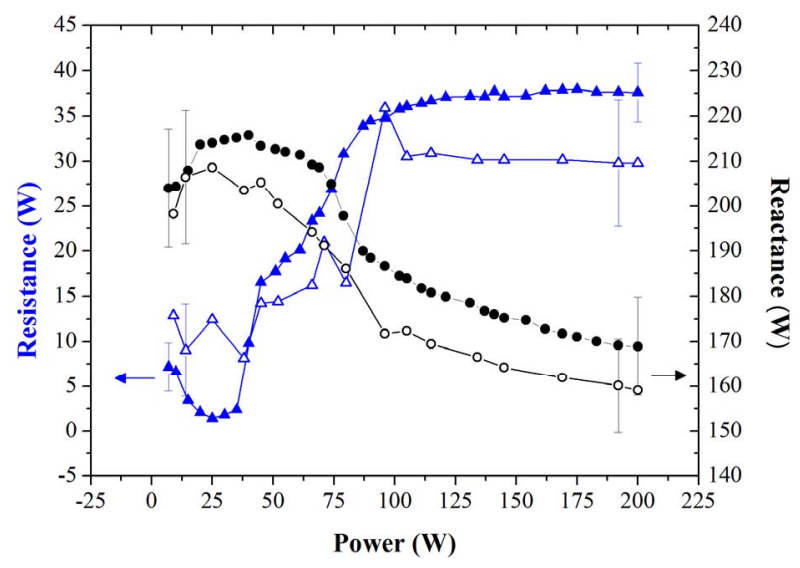

Figure 4. Real and imaginary part of the load impedance as a function of RF power in argon discharges at $40 \mathrm{~Pa}$.

procedures agree fairly well. The second being much more easy to employ was used in the subsequent experiments. Load resistance, which is dominated by the plasma contribution, starts to increase as the transition happens, and to stabilize in the H-mode. Load reactance, initially dominated by the antenna inductance, decreases as the plasma coupling becomes inductive, as expected from the simplified ICP model as a transformer [14]. A typical OES spectrum of argon discharges after dark subtraction and sensitivity correction is shown in Figure 5. The brightest feature in the spectra of such discharges is the system of $3 p^{5} 4 p \rightarrow 4 s$ transitions, the $2 p_{x}$ system in the Paschen notation, in the red and in the near IR [34]. Under vacuum conditions, the excitation of argon radiatively decaying levels happens mainly through inelastic scattering of argon atoms by high energy electron impact. Under such an approximation the intensity of emission lines is proportional to the product of the electron density and the rate of impact excitation process, which is a function of the reduced electric field or, in other terms, of the electron temperature alone [30]. We take advantage 


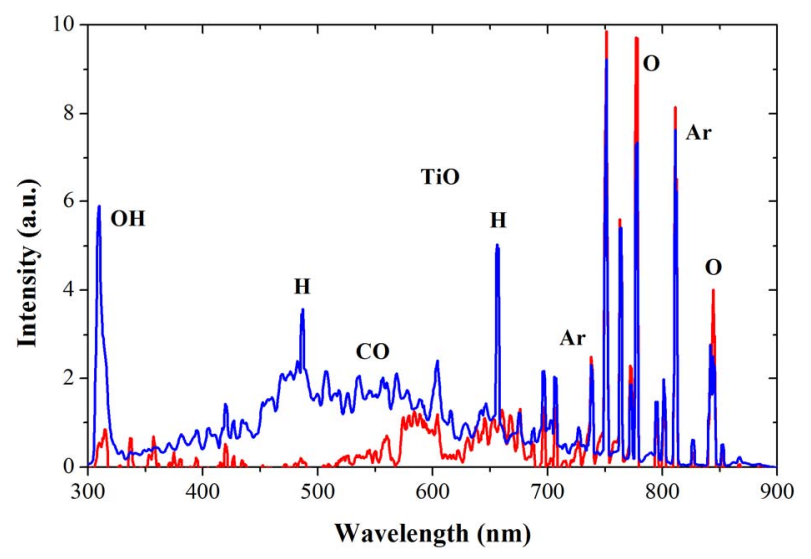

Figure 5. Typical spectra of the plasma before and after the TEOT precursor injection into the chamber.

of the presence of argon. The OES of argon plasmas is favourable in Visible and near-IR range and the dynamics of the excitation and radiative emission of the main energy levels is well known [35,36]. The relative intensity of some reference lines can so be used to gain insight mainly in the electron plasma properties. This was done for the $751 \mathrm{~nm}$ (emitted from the $2 \mathrm{p}_{5}$ level, mainly populated from the ground state argon [35]), which was one of the brightest in our OES spectra. Division by pressure factors out the effect due to the initial density of argon atoms, highlighting the contribution from the plasma electron alone. Results are shown in Figure 6 using a $\log$-scale. As already reported, the transition between $\mathrm{E}$ and H-mode in the OES intensity is sharp, at least at the highest pressures. The increase is so large that a log-scale is needed to appreciate features in both regimes. This is to be compared with the much more gentle transition displayed by electrical parameters discussed previously. In both regimes the intensity rises with the RF power level, although the rate is different and strongly dependent from pressure. This overall trend is common to the other emission lines of the $2 p_{\mathrm{x}}$ system, but their relative intensity changes slightly but significantly within the power and pressure ranges investigated. This reflects shifts in the electron excitation pattern influenced by changes in the electron temperature and the metastable argon abundances. Radiative models have been proposed in order to extract plasma parameters from the relative intensities of the emission line pattern in argon [37]. In particular a simplified model, including only the part of the $2 p_{x}$ system was proposed [38] and modified by us [39] to increase precision. As a results, from the relative intensities of argon emitting lines, it is possible to measure the electron temperature and the concentration of the two kinds of metastable argon atoms. Both measures are valuable in order to characterize the plasma dissociation capability and could also be implemented as a monitor control during the deposition process. Only a brief de-

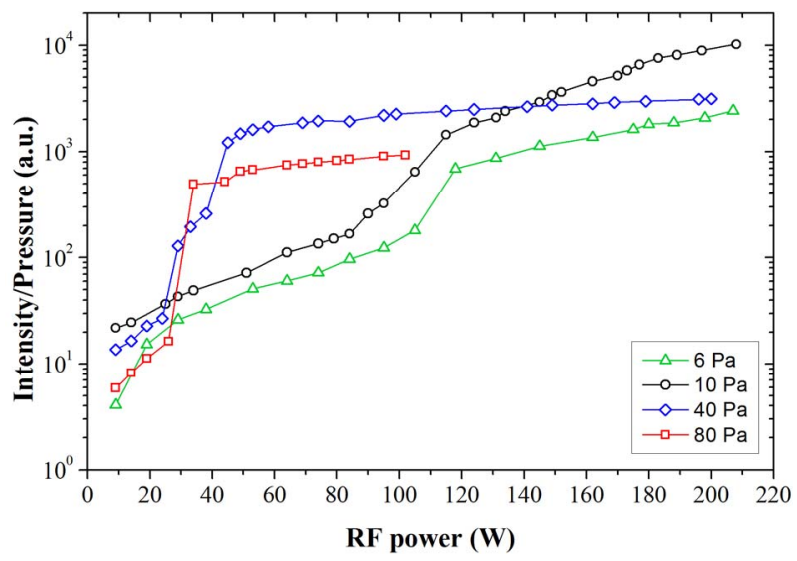

Figure 6. Intensity of $751 \mathrm{~nm}$ argon line normalised to the total Ar density as a function of the RF power.

scription of the model is provided here. The core of the method is to to exploit the different electron energy dependence of the cross section of some of the $2 p_{x}$ excited levels and the large differences in the probability of electron impact excitation of such levels starting from metastable argon atoms [35]. Our proposal was to use the intensities of the whole set of emission lines of the $2 p_{x}$ system, provided that their intensity is not negligible and that no ambiguity could arise (especially because of our limited wavelength resolution), to define a sort of $\chi^{2}$ function:

$$
\begin{gathered}
\chi^{2}=2 \cdot N \cdot(N-1) \sum_{n<m=1, N}\left[\log ^{2}\left(R_{n} / R_{m}\right)\right] \\
R_{n}=\left(I_{n} / b_{n}\right) /\left[k_{n}\left(T_{e}\right)\left(1-\sum x^{*}\right)+\sum k_{n}^{*}\left(T_{e}\right) x^{*}\right],
\end{gathered}
$$

where $I$ is the measured intensity of the selected emission line and $b$ is the branching ratio of that particular transition from the upper excited level involved. In the latter formula $k$ and $k^{*}$ are the electron impact collision rate to the excited level corresponding to the $\mathrm{n}$-th emission lines, respectively from the ground state and from the two metastable states, while $x^{*}$ are their concentrations. Minimization respect to the parameters allows to extract the electron temperature and the two metastable concentrations. As discussed in our published papers [39,40], this procedure is more sensible, reducing the systematic errors which could affect the proposed analytical solution [38]. Using the measured intensities of nine lines we got a fair reproduction of the relative intensity pattern, within $30 \%$, which corresponds to a $\chi^{2} \sim 0.1$. Results concerning this kind of characterization of the plasma state are displayed in Figure 7. Electron temperatures are almost constant, slightly decreasing with the power level, in the $\mathrm{H}$-mode. Their values stay in the $1 \div 1.5 \mathrm{eV}$ range. They are somewhat larger in the E-mode, however never exceeding $2 \mathrm{eV}$. Temperature decreases steadily as the pressure increases. It is clear that, despite being signifi- 


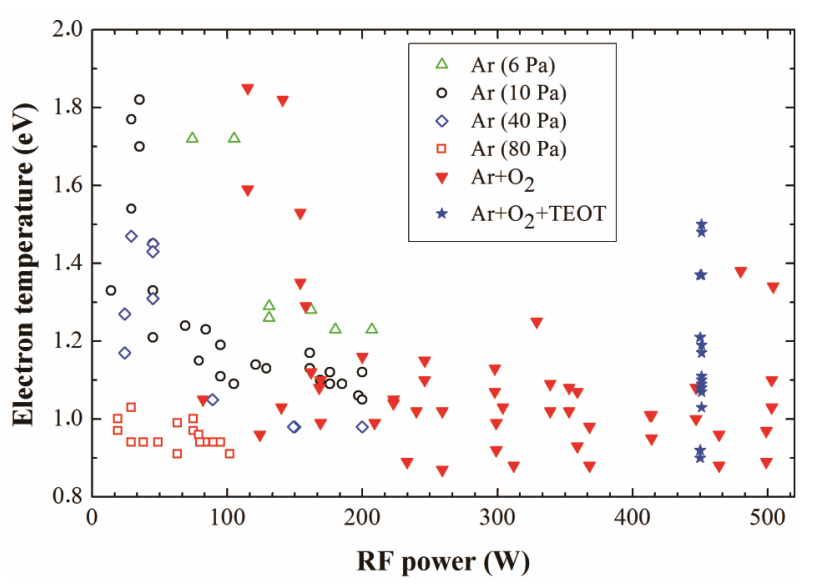

Figure 7. Electron temperature as a function of the RF power level in discharges of pure argon and mixtures.

cant, these differences cannot explain the sharp transition in the emission line intensity between the $\mathrm{E}$ and $\mathrm{H}$ modes. This is almost entirely due to a jump in the electron density in the $\mathrm{H}$ mode. Argon metastable atoms density was estimated in the range $10^{14} \div 10^{15} \mathrm{~m}^{-3}$, with concentrations around a ppm. Being similar, only slightly larger in the E mode, the concentration should not depend strongly on the electron density. This indicates that metastables are quenched mainly through electron impact or reactions with radicals itself produced by electron dissociation. When oxygen is added and diluted in argon, the qualitative behaviour of the source was similar. We have investigated an extended range of $\mathrm{O}_{2}$ concentrations from $5 \%$ to $80 \%$. In general a too high concentration of oxygen produces an increased stress on the pumping system. The addiction of oxygen has only a slight effect on the characteristics of the E-mode. However it delays the onset of the transition to higher power levels, between $100-200$ $\mathrm{W}$, and so voltage. Fully developed H-mode discharges often require power levels in excess of $500 \mathrm{~W}$, and voltage levels which continue to rise the larger is oxygen concentration. This could be guessed in data reported in Figure 8 with different argon/oxygen ratios at the same total pressure. The same trend could be observed also by keeping constant the argon partial pressure and increasing oxygen concentration. This behaviour is commonly ascribed to oxygen electronegativity, which favours the formation of negative ions, reducing the electron plasma density [19]. OES measurements confirm the overall picture. Oxygen molecules are poor emitting agents, discharge spectra in pure $\mathrm{O}_{2}$ being dominated by atomic and $\mathrm{O}_{2}^{+}$ion emission lines. The former are readily revealed also in our argon-oxygen ICP. Atomic argon and oxygen lines intensities show the same trend, with a strong increase at transition. The relative intensity decreases as oxygen concentration rises, in agreement with the decrease in the discharge current, at the same power level.

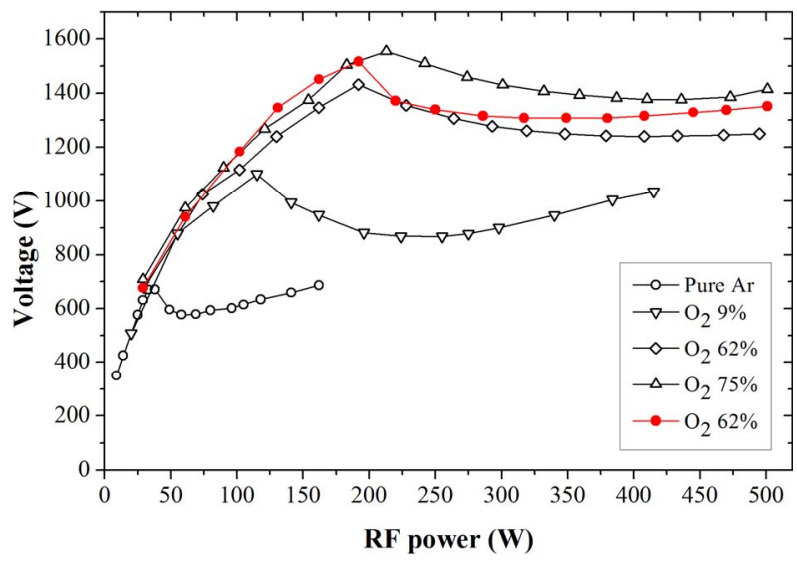

Figure 8. Coil HV amplitude as a function of the RF power in argon and mixtures at pressures about $10 \mathrm{~Pa}$.

Oxygen ion signal was spotted too, although being relatively weak and often undetectable, especially in the low power, low emitting E-mode. The presence of argon is favourable to OES in another way. Since it does not enter in any chemistry happening in the gas-phase, the intensity of its emission lines is controlled only by electron properties in the plasma state. Argon ground state concentration being quite precisely determined by argon partial pressure alone, which is easily measured and controlled. Ideally in a steady, uniform and Maxwellian plasma, by plasma density and temperature only. Then the ratio between the intensity of any emission line and that of one of the argon lines, generally nearby in wavelength, factors out the electron density, whose measure requires a plasma diagnostics. If the electron temperature variations are not large, a feature which in ICP is generally satisfied, the excitation rates are fixed and in the intensity ratio they introduce only a multiplicative factor. This procedure, usually applied with noble gases in trace and called actinometry [14], provides then a measure of the relative abundances of the emitting particles. This is already useful to compare dissociation capabilities of different plasma conditions. When the electron temperature and the dynamics of the excitation process is known, it is also possible to gain insight in the absolute concentration of the parent atoms or molecules, usually the ground state. In particular we minded the oxygen atom emission lines, which are easily detected in our spectra. The result of such an exercise is displayed in Figure 9, where the oxygen $777 \mathrm{~nm}$ emission line intensity was normalized against a pool of three nearby argon lines (750, 751 and $763 \mathrm{~nm}$ ). We measured also the 844 and $615 \mathrm{~nm}$ lines of oxygen, obtaining similar results. Although the former is usually preferred being the least influenced by indirect excitation channels [14], we found a partial contamination from the close $842.5 \mathrm{~nm}$ emission line of argon, due to the low resolution of the spectrometer. The intensity ratio, so the atomic oxygen density, increases steadily 


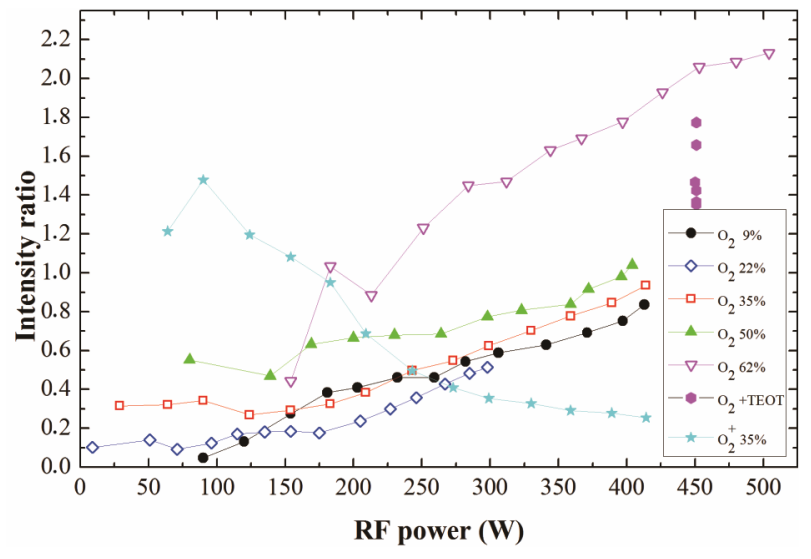

Figure 9. Intensity ratio between oxygen and argon lines as a function of the RF power, at the same argon pressure (10 Pa) with different $\mathrm{O}_{2}$ concentrations.

with the power level. It appears to change little whereas oxygen percentage in the gas mixture is between $10 \% \div$ $40 \%$, then it increases strongly. It could be observed that nevertheless the degree of dissociation of oxygen molecules in the $62 \%$ mixture is less than half of that in the $9 \%$ one. Molecular oxygen ion shows an opposite trend, decreasing with power and roughly proportional to the $\mathrm{O}_{2}$ percentage. It partially reflects the dissociation of the parent molecules, with a change in the charged species concentrations. This completes the characterization of the discharges in argon-oxygen mixtures in our device. However, since the source was developed to perform thin film deposition processes, we briefly describe the plasma state, when a precursor like TEOT is injected near the nozzle. We claim that by controlling plasma parameters it is possible to control the chemistry while by changing the pressure ratio $R$ and the distance of the substrate from the nozzle it is possible to tune nanoparticle size and energy and their deposition rate, controlling the whole film mesostructure. Deposition have been performed in a 450 W ICP discharge in a 2:3 $\mathrm{Ar} / \mathrm{O}_{2}$ mixture at $8 \mathrm{~Pa}$ pressure. TEOT heating was tuned to change precursor flowrate in order to find its optimal value. However in general the overall pressures in both the plasma and the deposition chamber do not change after precursor injection. Also the electrical behaviour is only slightly affected, both in the $\mathrm{E}$ and in H-mode. The same observation holds on even if the distance between the nozzle and the injection point is varied. In order to assess the precursor dissociation degree in the plasma state, it was important to monitor the relative emission line intensities of other emitting levels, produced in different stages of the precursor chemical kinetics evolution. In particular, due to the extended range of wavelength of our measured spectra, it was possible to spot the emission of several molecules produced by the chemical kinetics of TEOT in the plasma gas phase (see Figure 5). When the precursor is injected the emission spectrum changes substantially, showing evidences of a continuum and of several new molecules. Signals attributable to carbon oxide, $\mathrm{CH}$ and $\mathrm{OH}$ radicals and hydrogen atoms are readily observed in the OES spectra. In particular the carbon oxide is thought to be directly related to the degree of oxidation of the organic groups of TEOT. This is mirrored by the relative decline of $\mathrm{O}$ atoms emission which reveals TEOT oxidation by such plasma radicals (see Figure 9). Even more interesting was the emission attributable to $\mathrm{TiO}$ radicals [41] around $615 \mathrm{~nm}$. We also noticed the absence of direct emission from $\mathrm{Ti}$ atoms, which could be taken as a signal that dissociation stage was let evolve too much and so used as a monitor to discard operating conditions probably unfavourable for $\mathrm{TiO}_{\mathrm{x}}$ thin film deposition. Argon presence could be used again as an actinometry agent, to measure the relative abundances of radicals. A few results are shown in Figure 10, which refers to experiments aimed to study the effect of the precursor heating temperature, which controls the flow of TEOT. Increasing heating results in a generalized rise of radical emission relatively to argon atoms which are produced by dissociation and oxidation in the plasma gas-phase. However, by considering the different pace of the rise, we could grasp a relative decrease in the ratio of $\mathrm{CO}$ (and $\mathrm{OH})$ over the $\mathrm{H}$ atom. This suggests an incomplete oxidation of the organic groups of the precursor at high fluxes. This is also correlated to a relative decrease of $\mathrm{TiO}$ abundance at the highest temperatures, respect to both $\mathrm{H}$ atoms and $\mathrm{OH} / \mathrm{CO}$ molecules. The data could then be used to optimize the precursor flow and the discharge power level, as well as the distance between the injection needle and the nozzle. They were also correlated with an X-rays analysis (EDX) showing that at the highest temperatures the film is composed mainly by metal titanium. The oxygen titanium ratio increases by reducing the TEOT heating, reaching the optimal value around 2 when the temperature was about $120^{\circ} \mathrm{C} \div 130^{\circ} \mathrm{C}$. This demonstrates that suitable flowrate of precursor and chemical composition of the supersonic jet could be somewhat controlled. Here, however, spatially resolved measurements, to be taken by optical fibers placed directly in the vacuum chamber, will be valuable. Using SEM imaging it was possible to investigate the structure of the thin film and to measure its depth. This goes beyond the scope of our paper. However a few observations are worthy. In particular, compact thin films can be obtained when deposition occurs at high $R$ values (generally larger than 10) and in the expansion plume region, at deposition rates of about $200 \mathrm{~nm} / \mathrm{min}$. A more extended characterization of the deposited thin film will be presented elsewhere [17]. Film composition could be assessed too, by using EDX. The kind of results obtained through such analysis is shown in Figure 11. Here depo- 


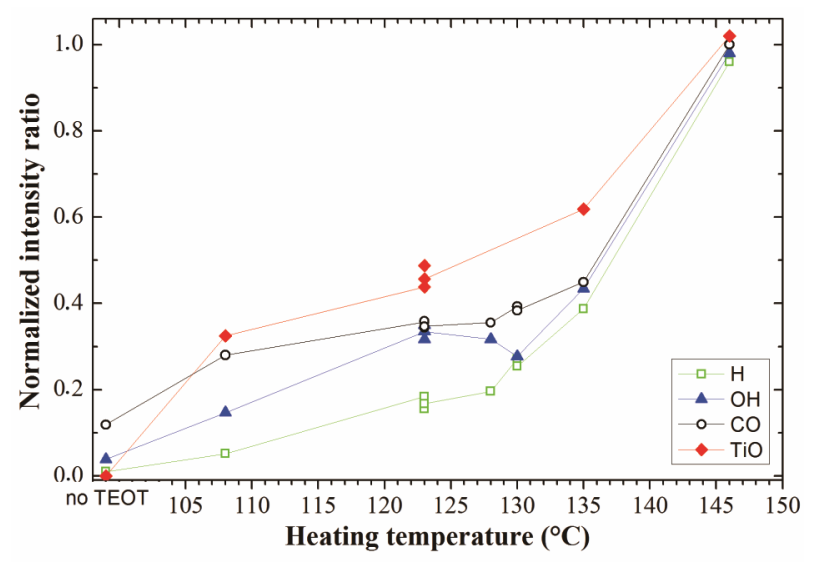

Figure 10. Intensity ratio between $\mathrm{TiO}, \mathrm{CO}, \mathrm{OH}, \mathrm{H}$ and argon lines as a function of TEOT heating temperature.

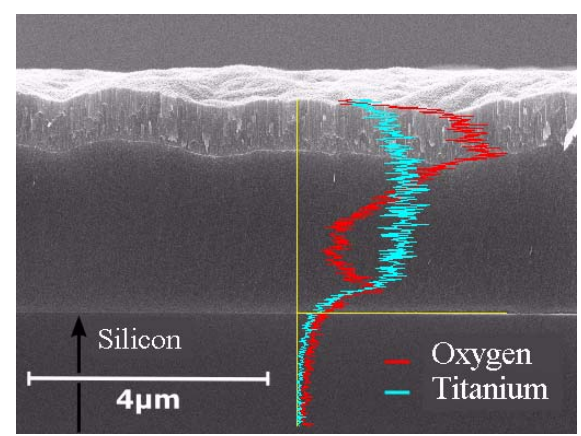

Figure 11. SEM image of a thin film section deposited from TEOT on a silicon substrate.

sition was started at a the precursor flowrate which was changed afterwards. As shown in Figure 11, uniform, compact and quite thick film could be deposited in a relatively short time. The relative EDX signals of oxygen and titanium are reported as a function of the film depth. While, at first, an almost metal titanium film was obtained, then a quite stechiometric $\mathrm{TiO}_{2}$ composition is achieved. So, in particular under the latter operating conditions, high deposition rates and pure $\mathrm{TiO}_{2}$ films can be easily obtained. We only add that, while the deposited film is almost amorphous, positive indications of film crystallization were obtained after annealing process. As a matter of fact, the RAMAN spectra of the annealed film showed an almost complete transformation in anatase. The same can be assessed from Figure 12, which displays the angle resolved X-ray diffraction pattern of our $\mathrm{TiO}_{2}$ nanostructured thin film. The peaks at scattering angles around $25^{\circ}, 38^{\circ}$ and $48^{\circ}$ correspond to the reflections from some crystal planes of anatase. No crystalline phase assignable to rutile and brookite could be found. Then, by changing the pressure ratio $\mathrm{R}$ and the substrate location, our source was shown to be able to successfully deposit titanium oxide films with thickness from hundreds of nanometers up to a few microns.

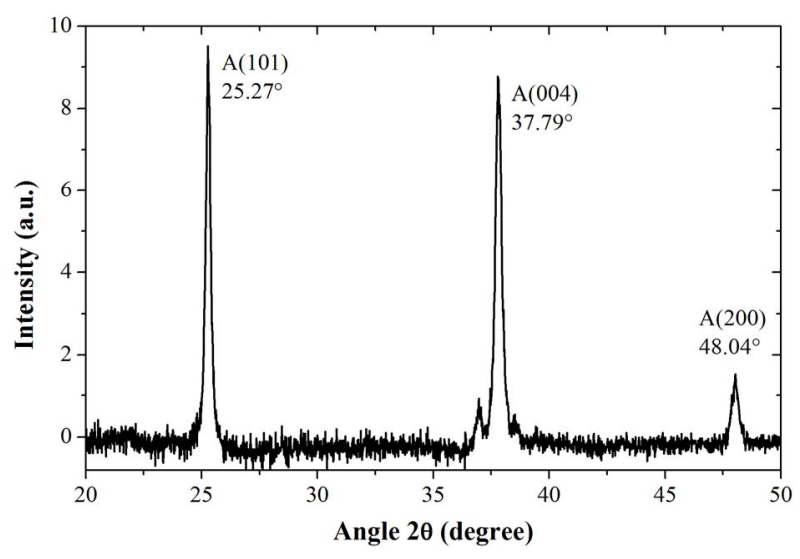

Figure 12. XRD of $\mathrm{TiO}_{2}$ thin film after annealing showing the characteristic peaks of anatase allotropic phase, marked with letter $\mathrm{A}$ and the corresponding scattering angles.

\section{Conclusion}

A new plasma source for thin film deposition was presented and characterized. It embodies the idea of a spatial separation between the precursor dissociation, to be performed in a plasma environment, and the thin film growth, to be performed on a substrate watered by a flow of molecular clusters, which evolve and aggregate during the transfer. Here a supersonic jet substantially free expansion was believed to be the better environment to allow control of cluster sizes and flowrate. Plasma characterization has been performed, demonstrating the capability of the source to maintain stable ICP for long periods, with flexible and controllable parameters that could be tuned for an optimization of the precursor dissociation. Feasibility of thin film titanium oxide deposition, with suitable growth rate and hardness and controlled composition, was demonstrated using a standard precursor like TEOT. Then, by changing the pressure ratio $R$ and the substrate location, the flowrate, the size and the energy of precursor clusters can be varied and so the thin film morphology could be controlled too.

\section{Acknowledgements}

Part of the work was performed under a grant by Fondazione Cariplo (Scientific and Technological Research on Advanced Materials-2010, nr. 2010.0623), an Italian private funding agency, whose support we gratefully acknowledge. We are also pleased to thank technical and scientific collaborators of the PlasmaPrometeo Center for the living and stimulating atmosphere of collaboration.

\section{REFERENCES}

[1] K. J. Seshan, "Handbook of Thin-Film Deposition Processes and Techniques," W. Andrew Publishing, Noyes, 2002. 
[2] S. Ramanathan, "Thin Film Metal-Oxides," Springer, Heidelberg, 2010. doi:10.1007/978-1-4419-0664-9

[3] M. Graetzel, "Sol-Gel Processed $\mathrm{TiO}_{2}$ Films for Photovoltaic Applications," Sol-Gel Science and Technology, Vol. 22, No. 1-2, 2001, pp. 7-13. doi:10.1023/A:1011273700573

[4] J. Aguado-Serrano and M. L. Rojas-Cervantes, "Titania Aerogels: Influence of Synthesis Parameters on Textural, Crystalline and Surface Acid Properties," Microporous and Mesoporous Materials, Vol. 88, No. 1-3, 2006, pp. 205-213. doi:10.1016/j.micromeso.2005.09.011

[5] X. C. Wang, J. C. Yu, C. M. Ho, Y. D. Hou and X. Z. Fu, "Photocatalitic Activity of a Hierarchically Macro/Mesoporous Titania," Langmuir, Vol. 21, No. 6, 2005, pp. 2552-2559. doi:10.1021/la047979c

[6] M. Suzuki, T. Ito and Y. Taga, "Photocatalysis of Sculptured Thin Films of $\mathrm{TiO}_{2}$," Applied Physics Letters, Vol. 78, No. 25, 2001, pp. 3968-3970. doi: $10.1063 / 1.1380730$

[7] S. M. Waita, B. O. Aduda, J. M. Mwabora, C. G. Granqvist, S. E. Lindquist, G. A. Niklasson, A. Hafeldt and G. Boschloo, "Electron Transport and Recombination in Dye Sensitized Solar Cells Fabricated from Obliquely Sputter Deposited and Thermally Annealed $\mathrm{TiO}_{2}$ Films," Journal of Electroanalytical Chemistry, Vol. 605, No. 2, 2007, pp. 151-156. doi:10.1016/j.jelechem.2007.04.001

[8] J. Rodriguez, M. Gomez, J. Lu, E. Olsson and C. G. Granqvist, "Reactively Sputter-Deposited Titanium Oxide Coatings with Parallel Penniform Microstructure," Advanced Materials, Vol. 12, No. 5, 2000, pp. 341-343. doi:10.1002/(SICI)1521-4095(200003)12:5<341::AID-A DMA341>3.0.CO;2-0

[9] F. Di Fonzo, C. S. Casari, V. Russo, M. F. Brunella, A. L. Bassi and C. E. Bottani, "Hierarchically Organized Nanostructured $\mathrm{TiO}_{2}$ for Photocatalysis Applications," Nanotechnology, Vol. 20, No. 1, 2009, pp. 1-7.

[10] A. Goossens, E. L. Maloney and J. Schoonman, "GasPhase Synthesis of Nanostructured Anatase $\mathrm{TiO}_{2}$," Chemical Vapor Deposition, Vol. 4, No. 3, 1998, pp. 109114.

doi:10.1002/(SICI)1521-3862(199805)04:03<109::AID-C VDE109>3.0.CO;2-U

[11] L. Mangolini, E. Thimsen and U. Kortshagen, "High-Yield Plasma Synthesis of Luminescent Silicon Nanocrystals," Nanoletters, Vol. 5, No. 4, 2005, pp. 655-659. doi:10.1021/n1050066y

[12] H. Nizard, M. L. Kosinova, N. I. Fainer, Y. M. Rumyantsev, B. M. Ayupov and Y. V. Shubin, "Deposition of $\mathrm{TiO}_{2}$ from TTIP by Plasma and Remote Plasma Enhanced CVD," Surface and Coatings Technology, Vol. 202, No. 17, 2008, pp. 4076-4085. doi:10.1016/j.surfcoat.2008.02.023

[13] J. Hopwood, "Review of Inductively Coupled Plasmas for Plasma Processing," Plasma Sources Science and Technology, Vol. 1, No. 2, 1992, pp. 109-116. doi:10.1088/0963-0252/1/2/006

[14] M. A. Lieberman and A. J. Lichtenberg, "Principles of Plasma Discharges and Materials Processing," Wiley,
New York, 1998.

[15] R. Siliprandi, S. Zanini, E. Grimoldi, F. Fumagalli, R. Barni and C. Riccardi, "Atmospheric Pressure Plasma Discharge for Polysiloxane Thin Films Deposition and Comparison with Low Pressure Process," Plasma Chemistry and Plasma Processing, Vol. 31, No. 2, 2011, pp. 353-372. doi:10.1007/s11090-011-9286-3

[16] S. F. Durrant, N. C. Da Cruz, E. C. Rangel and M. A. Bica de Moraes, "Plasma Enhanced Chemical Vapor Deposition of Titanium (IV) Ethoxide-Oxygen-Helium Mixtures," Thin Solid Films, Vol. 516, No. 15, 2008, pp. 4940-4945. doi:10.1016/j.tsf.2007.09.036

[17] C. Riccardi, I. Biganzoli and R. Barni, "Experimental Characterization of a New Plasma Source for Controlled Deposition of Thin Films," Europhysics Conference Abstracts, Vol. 36F, 2012, pp. 1-4.

[18] P. H. Oosthuizen and W. E. Carscallen, "Compressible Fluid Flow,” McGraw-Hill, London, 1997.

[19] J. T. Gudmmundsson, T. Kimura and M. A. Lieberman, "Experimental Studies of $\mathrm{O}_{2} / \mathrm{Ar}$ Plasma in a Planar Inductive Discharge," Plasma Sources Science and Technology, Vol. 8, No. 1, 1999, pp. 22-30. doi:10.1088/0963-0252/8/1/003

[20] J. Kowalski, A. Sobczyk-Guzenda, H. Szymanowski and M. Gazicki-Lipman, "Optical Properties and Morphology of PECVD Deposited Titanium Dioxide Films," Journal of Achievements in Materials and Manufacturing Engineering, Vol. 37, No. 2, 2009, pp. 298-303.

[21] K. Jousten, "Handbook of Vacuum Technology," Wiley, New York, 2008.

[22] J. M. Smith, "Introduction to Chemical Engineering Thermodynamics," McGraw-Hill, New York, 1987.

[23] F. Sharipov and D. V. Kozak, "Rarefied Gas Flow through a Thin Slit at an Arbitrary Pressure Ratio," European Journal of Mechanics B, Vol. 30, No. 5, 2011, pp. 543549. doi:10.1016/j.euromechflu.2011.05.004

[24] L. Holland, W. Steckelmacher and J. Yarwood, "Vacuum Manual,” E.\&F.N. Spon Ltd., London, 1974. doi:10.1007/978-94-011-8120-4

[25] H. R. Murphy and D. R. Miller, "Effects of Nozzle Geometry on Kinetics in Free-Jet Expansions," Journal of Physical Chemistry, Vol. 88, No. 20, 1984, pp. 44744478. doi:10.1021/j150664a005

[26] H. Ashkenas and F. Sherman, "Structure and Utilization of Supersonic Free Jets in Low Density Wind Tunnels," In: Rarefied Gasdynamics 4, Academic Press, New York, 1966.

[27] C. Raju and J. Kurian, "Effect of Slit Aspect Ratio on Free Jet Properties," Vacuum, Vol. 46, No. 4, 1995, pp. 389-395. doi:10.1016/0042-207X(94)00085-9

[28] Y. Otobe, H. Kashimura, S. Matsuo, T. Setoguchi and H. D. Kim, "Influence of Nozzle Geometry on the NearField Structure of a Highly Underexpanded Sonic Jet," Journal of Fluids and Structures, Vol. 24, No. 2, 2008, pp. 281-293. doi:10.1016/j.jfluidstructs.2007.07.003

[29] R. A. Siliprandi, H. E. Roman, R. Barni and C. Riccardi, "Characterization of the Streamer Regime in Dielectric 
Barrier Discharges," Journal of Applied Physics, Vol. 104, No. 6, 2008, pp. 1-9. doi:10.1063/1.2978184

[30] V. M. Donnelly, "Plasma Electron Temperatures and Electron Energy Distributions Measured by Trace Rare Gases OES," Journal of Physics D, Vol. 37, No. 19, 2004, pp. R217-R236. doi:10.1088/0022-3727/37/19/R01

[31] U. Kortshagen, N. D. Gibson and J. E. Lawler, "On the E-H Mode Transition in RF Inductive Discharges," Journal of Physics D, Vol. 29, No. 5, 1996, pp. 1224-1236.

[32] F. Croccolo, A. Quintini, R. Barni and C. Riccardi, "Transition between E-Mode and H-Mode in a Cylindrical ICP Reactor," High Temperature Material Processes, Vol. 14, No. 1-2, 2010, pp. 119-127.

[33] C. M. Tsai, A. P. Lee and C. S. Kou, "Characteristics of Heating Mode Transitions in a RF Inductively Coupled Plasma," Journal of Physics D, Vol. 39, No. 17, 2006, pp. 3821-3825.

[34] J. E. Chilton, J. B. Boffard, R. S. Schappe and C. C. Lin, "Measurement of Electron-Impact Excitation into the $3 p^{5} 4 p$ Levels of Argon Using Fourier-Transform Spectroscopy," Physical Review A, Vol. 57, No. 1, 1998, pp. 267-277.

[35] A. Dasgupta, M. Blaha and J. L. Giuliani, "Electron-Im- pact Excitation from the Ground and the Metastable Levels of Ar I," Physical Review A, Vol. 61, No. 1, 2000, pp. $1-10$.

[36] Y. Ralchenko, A. Kramida, J. Reader and NIST-ASD Team, "NIST Atomic Spectra Database," 2011. http://physics.nist.gov/asd

[37] J. Vlcek, "Collisional-Radiative Model Applicable to Argon Discharges over a Wide Range of Conditions," Journal of Physics D, Vol. 22, No. 5, 1989, pp. 623-643.

[38] D. Mariotti, Y. Shimizu, T. Sasaki and N. Koshizaki, "Method to Determine Argon Metastable Number Density and Plasma Electron Temperature from Spectral Emission Originating from Four 4p Argon Levels," $A p$ plied Physics Letters, Vol. 89, No. 20, 2006, pp. 1-4.

[39] F. Croccolo, R. Barni, S. Zanini, A. Palvarini and C. Riccardi, "Material Surface Modifications with an Inductive Plasma," Journal of Physics: Conference Series, Vol. 100, No. 6, 2008, pp. 1-3.

[40] R. Barni, S. Zanini and C. Riccardi, "Diagnostics of RF Reactive Plasmas," Vacuum, Vol. 82, No. 2, 2007, pp. 217-219.

[41] R. W. B. Pearse and A. G. Gaydon, "The Identification of Molecular Spectra,” Wiley, New York, 1976. 